\title{
Gestión Educativa Municipal: Desafíos para una Educación de Calidad en Chile
}

\author{
María del Pilar Laso Correa ${ }^{37}$ \\ Manuel Albarrán Ulsen ${ }^{38}$ \\ Natacha Carrasco Salinas ${ }^{39}$ \\ Chile
}

Fecha recepción: $13 / 0 \mid / 2012$

Fecha aceptación: 20/03/2012

\section{Resumen}

La calidad de la Educación que reciben los niños en Chile está condicionada a su nivel socioeconômico, lo que genera una situación de inequidad y falta de igualdad de oportunidades para los más vulnerables, en el cual la educación pública, impartida a través de los Municipios, tiene un rol relevante toda vez que concentra la mayor cantidad de alumnos desfavorecidos.

En este trabajo se presentan los resultados del estudio de percepción sobre la gestión y funcionamiento en las Direcciones de Administración en Educación Municipal (DAEM $)^{40}$ de la Región del Biobío, y su implicancia en los problemas de mejoramiento de la calidad de la Educación en Chile. Se visualiza aquí la complejidad en las normativas y roles que deben cumplir compatibilizando su gestión administrativa con educacional orientada a la calidad.

Con esto se espera contribuir al sistema educacional, compartiendo las problemáticas de gestión en las Direcciones de Administración de Educación Municipal (DAEM) que es posible abordar, fortaleciendo su rol como sostenedores, enfatizando en lo pedagógico y en la medición de los resultados de aprendizaje de sus alumnos.

Palabras claves: Calidad de la educación pública - Gestión Educacional - Equidad Educativa

37 Coordinadora del Área de Educación y Desarrollo del Programa de Políticas Públicas y Ciudadanía de la Universidad del Bío-Bío, Profesora de Educación Diferencial con mención en Trastornos del Aprendizaje, Mg. Gestión, Liderazgo y Política Educativa.

38 Director del Programa de Políticas Públicas y Ciudadanía de la Universidad del Bío-Bío,Licenciado Bioquímico,Magíster en Investigación Social y Desarrollo,

39 Investigador de Apoyo, Socióloga, Magíster en Política y Gobierno .

40 La denominación de estas reparticiones presenta variaciones en los distintos Municipios, pero es reconocida como Dirección de Administración de Educación Municipal en la mayoría de ellos, con la abreviarura DAEM la cual se utiliza en este documento 


\title{
Municipal Education Management: Challenges for a Quality Education in Chile.
}

\begin{abstract}
The quality of the education that the children receive in Chile, is was determined to his level economic partner generating a situation of inequity and lack of equality of opportunities for the more vulnerable persons, in which the public education, given by the Municipalities, has a relevant role although it concentrates the major quantity of disadvantaged pupils.

This paper present the results of the study of perception on the management and functioning in the Directions of Municipal Education of the VIII Region, and Its implication in the problems of improvement of the quality of the education in Chile. In this is visualized the complexity in the regulations and roles that must expire making compatible his administrative management with educationally orientated to the quality.

With this one expects to contribute educational system, sharing the problematic ones of management in the DAEM that it is possible to approach, strengthening his role as administration emphasizing the pedagogic thing and the measurement of the results of learning of his pupils.
\end{abstract}

Key words: Quality public education - Management and functions education Municipal education

\section{Gestão educativa municipal: Desafios para uma educação de qualidade no Chile.}

\section{Resumo}

A qualidade da educação que as crianças recebem no Chile está condicionada ao seu nível socioeconômico, o que gera uma situação de injustiça e desigualdade de oportunidades para os mais vulneráveis; situação da qual a educação pública oferecida por meio dos municípios tem um papel relevante, uma vez que ela concentra a maior quantidade de alunos desfavorecidos.

Neste trabalho se apresentam os resultados do estudo de percepção sobre a gestão e funcionamento nas direcciones de administracióneneducación municipal (DAEM)da região de Biobíoe sua implicância nos problemas de melhoramento da qualidade da educação no Chile.Visualiza-se aqui a complexidade das normas e papéis que devem cumprir as DAEM, compatibilizando sua gestão administrativa com a educacional, orientada à qualidade. 
Com este estudo se espera poder contribuir com o melhoramento do sistema educacional, ao compartir as problemáticas de gestão nas DAEM possíveis de serem abordadasecomo fortalecer seu papel sustentador, enfatizando o pedagógico e a medição dos resultados de aprendizagem dos seus alunos.

Palavras-chaves: Qualidade da educação - Gestão da educação - Educação unicipalizada - Sustentadores da Educação.

\section{INTRODUCCIÓN}

La baja calidad de la educación en Chile constituye una preocupación central en la sociedad actual, ya que los resultados obtenidos por niños y jóvenes en pruebas estandarizadas como Simce, PISA, TIMSS, PSU, son menores a los esperados para nuestro nivel de desarrollo. Complementariamente los estudios internacionales como "Revisión económica: Chile 2010", realizado por la OCDE, indican que mejorar los estándares de calidad de la educación es fundamental para poder aspirar a nuevos estándares de desarrollo.

Al realizar un análisis respecto de la calidad de la Educación es posible observar que si bien afecta a todos los sectores por igual (resultados PISA ${ }^{41}$ y TIMSS $^{42}$ ), es en los sectores socioeconómicos más bajos (quintil I y 2 de ingresos) donde el problema hace crisis, ya que más del $80 \%$ de los alumnos no logra obtener los aprendizajes básicos esperados para su nivel escolar.

Si bien esta situación deriva de múltiples factores, una constatación es que el $70 \%$ de los alumnos más pobres asiste a colegios públicos, en los cuales el sostenedor es el Municipio a través de las Direcciones de Administración de Educación Municipal.

Por esto, el presente estudio, desarrollado por el Programa de Políticas Públicas y Ciudadanía de la Universidad del Bío-Bío, mediante su área de Educación y Desarrollo; se propuso analizar la gestión y funcionamiento de las Direcciones de Educación Municipal de la Región del Biobío, con relación a los problemas de mejoramiento de la calidad de la educación y, a su vez, indagar sobre las características de estos sostenedores para dar solución a problemas estructurales y funcionales de la educación en su comuna. Con este trabajo se espera hacer una contribución al estudio y definición de las políticas públicas orientadas al mejoramiento de la calidad y equidad de la educación en Chile.

Este corresponde a un estudio descriptivo de tipo exploratorio, que pretende identificar percepciones, conceptos y variables relevantes, con un esquema metodológico cualitativo, basado en la realización de entrevistas semi estructuradas a actores claves (Directores) de los DAEM de la Región del Biobío, compuesta

4I Programme of international Sutdent assessment (PISA)

42 Trends in International Mathematics and Science Study (TIMSS). 
de 12 preguntas abiertas, orientadas a conocer percepciones sobre la gestión Educativa. El instrumentos fue construido a partir de los criterios considerados en los sitemas de gestion de la calidad de la gestion escolar sobre liderazgo y gestion estratégica, en especial del Sistema de Aseguramiento de la Calidad Escolar ( $S A C G E)$ del Mineduc.

Así, durante el primer semestre del año 20I I se entrevistó en municipios seleccionados, sobre un muestreo intencional nominativo, seleccionado bajo criterios de accesibilidad y pertinencia como la distribución territorial, su configuración urbana o rural y el tamaño de las comunas según cantidad de habitantes. La muestra quedó conformada por ocho Directores de DAEM de comunas de la Región del Biobío: Concepción, Talcahuano, San Pedro de la Paz, Hualqui, Florida, Cabrero, Coelemu y San Nicolás.

La información recopilada fue transcrita, preprocesada y revisados los antecedentes secundarios, tras lo cual se construyó una matriz temática con el análisis de elementos como misión, visión, gestión del DAEM, metas y objetivos, problemas visualizados y soluciones propuestas.

\begin{tabular}{|l|l|l|}
\hline \multirow{2}{*}{ Ámbito } & \multicolumn{1}{|c|}{ Tema } & Datos \\
\hline \multirow{4}{*}{ Gestión DAEM } & Misión & \\
\hline & Visión & \\
\cline { 2 - 3 } & Gestión & \\
\cline { 2 - 3 } & Metas y objetivos & \\
\hline & Problemas & \\
\hline & Soluciones & \\
\hline
\end{tabular}

Luego se procedió a la transcripción y análisis de los datos recopilados, elaborando una pauta de codificación, basada en matrices temáticas, mediante la cual se fueron clasificando las respuestas según tópicos previamente definidos en la investigación. Con la información organizada, se realizó un análisis de contenido, según los discursos entregados por los entrevistados, de los cuales derivaron criterios únicos y transversales, los que fueron abordados de manera específica para el resultado de la investigación.

Este artículo busca contribuir al sistema educacional nacional, compartiendo con el resto de instituciones la percepción de directores de DAEM sobre su gestión como sostenedor para presentar un servicio educativo de calidad. Así también presenta algunas de las problemáticas y debilidades en la gestión de estas unidades que deberían ser consideradas para avanzar hacia una educación pública con equidad y calidad.

\section{PRINCIPALES POLÍTICAS DE CALIDAD DE LA EDUCACIÓN EN CHILE}

\section{a. Contexto Nacional}

Al inicio de los 80, en Chile, se implementó un conjunto de modificaciones legales que llevaron a la Reforma en Educación, la cual ha tenido grandes implicancias en 
la calidad y equidad de la educación. ${ }^{43}$ Este cambio instaló, entre otras medidas, la posibilidad de entregar subvención pública a sostenedores privados ${ }^{44}$, es decir, organizaciones con 0 sin fines de lucro para que pudiesen entregar servicios de educación con financiamiento público. Complementariamente la política de descentralización entregó a los municipios atribuciones para hacerse cargo de la administración de las escuelas públicas ${ }^{45}$, esto, con la finalidad de revertir la inequidad, las altas tasas de repetición y deserción de los alumnos del sistema escolar existentes en Chile al inicio de los años 80 .

Estas medidas permitieron la expansión de la cobertura en educación, que en 1980 era de un $89.4 \%$ en básica y de un 79\% en media, llegando el año 2006 a un total de $99.8 \%$ de acceso en básica y de un $96 \%$ en Media.

El crecimiento de la matrícula fue facilitado, en gran medida, por aumento de establecimientos educacionales particulares subvencionados, administrados por sostenedores privados, que generaron nuevas vacantes en el sistema y capturaron al nivel económico medio, logrando en los últimos 20 años el 45, 3\% de la matrícula total de alumnos de enseñanza básica, reduciento la matrícula del sector municipal a un $48,2 \%$; en tanto que el sector particular pagado se ha mantenido con el $6,5 \%$ de los estudiantes ${ }^{46}$.

Esta expansión en la cobertura educacional impacta principalmente en los primeros dos quintiles de ingreso de la población, es decir, a los sectores más pobres (Marcel y Tokman, 2005.), permitiendo su ingreso al sistema. Sin embargo, su progresividad se encuentra condicionada a la calidad de la educación que reciben, vinculada a su condición socioeconómica. Si bien hoy la cobertura de matrícula en los distintos niveles de la educación escolar es similar en los diversos estratos económicos, lo que aprenden los niños es diferente dependiendo de su condición social, lo que finalmente determina su acceso a la educación superior y, por tanto, el nivel de ingresos al que puede optar a futuro

Según los autores Michael y Mourshed (2008), los niveles de calidad, equidad y eficiencia en el sistema de educación siguen siendo bajos y el progreso escaso o nulo, por lo que la posibilidad de los niños latinoamericanos, especialmente los más pobres, de acceder a los conocimientos y competencias necesarias para el éxito

43 Constitución Política de la República, aprobada en 1980, que considera diversas disposiciones generales que inciden sobre la organización y gestión del sistema educacional, como las relativas a la administración del Estado, a la descentralización y a la propiedad privada y la libre gestión de las empresas. Esta fue complementada con la Ley Orgánica Constitucional de Enseñanza, N 18.962.

44 Ley de Subvenciones Educacionales, dictada en 1980, normativa sobre el subsidio estatal a la educación particular subvencionada y a los establecimientos bajo administración municipal.

45 Decreto Ley de Rentas Municipales, $N^{\circ} 3.063$, de 29 de diciembre de 1979 , que, en su artículo $38^{\circ}$, facultó al gobierno para traspasar servicios desde la administración central del Estado a las municipalidades, entre ellos, los establecimientos educacionales que dependían del Ministerio de Educación.

46 Datos Generales Ministerio de Educación on line: http://www.mineduc.cl/index2.php?id_contenido=| |499\&id_ portal=17\&id_seccion $=2893$ 
de los países desarrollados, se ve obstaculizada por los bajos niveles de aprendizaje (especialmente en las áreas de ciencias y lenguaje), la falta de sistemas de gestión de resultados, $y$ un deficitario ejercicio docente.

Esta situación educacional, con baja calidad y alta desigualdad, no es sostenible en el tiempo, considerando que inhibe la movilidad e integración social y que promueve la generación de una fuerza de trabajo de baja calidad, limitando las posibilidades de desarrollo y crecimiento económico (Contreras, Lagraña, Flores, Lobato y Macías, 2003).

Antes de la aplicación de la política de extensión de cobertura, los sectores sociales más postergados o pobres no tenían mayores oportunidades de acceder a la educación, tanto básica como media y superior, por lo que los sistemas educacionales funcionaban, en general, sin mayores sobresaltos gracias al capital cultural que traen los alumnos consigo desde sus hogares. Sin embargo, hoy tenemos un cambio radical en las características de los alumnos, específicamente en los que asisten al sistema público, provenientes de niveles socioeconómicos de alta vulnerabilidad, económica y cultural, que han hecho evidente las deficiencias de los sistemas educacionales para trabajar en forma efectiva con los sectores más vulnerables, quienes reciben una educación de menor calidad, traducida en menores logros de aprendizajes.

\section{b. Concepto de calidad en Educación}

La OCDE (Organisation for Economic Co-operation and Development) define la educación de calidad como aquella que "asegura a todos los jóvenes la adquisición de los conocimientos, capacidades, destrezas y actitudes necesarias para equipararles para la vida adulta", en tanto que Bravlasky (2004) sostiene que una educación de calidad es aquella que permite que todos aprendan lo que necesitan aprender, en el momento oportuno de su vida y de sus sociedades y en felicidad. ${ }^{47}$

El significado atribuido a la expresión "calidad de la educación" incluye varias dimensiones o enfoques, complementarios entre sí. Elola y Toranzos (2000) sostienen que, en el ámbito educativo, la calidad puede considerarse en al menos tres dimensiones.

En un primer sentido el concepto de calidad puede ser entendido como eficacia: una educación de calidad sería aquella que logra que los alumnos realmente aprendan lo que se supone deben aprender al cabo de determinados ciclos o niveles. Esta dimensión del concepto pone en primer plano los resultados de aprendizaje efectivamente alcanzados por la acción educativa.

47 Citado en:Jiménez, Jorge. Mejorando la calidad de la enseñanza y el aprendizaje para todos los estudiantes. On line: http://www.emol.com/educacion/programas/educadores20/pdf20l0/jimenez.pdf 
Una segunda dimensión del concepto de calidad está referida a qué es lo que se aprende en el sistema y a su relevancia en términos individuales y sociales. En este sentido una educación de calidad sería aquella cuyos contenidos responden adecuadamente a lo que el individuo necesita para desarrollarse como persona y para desempeñarse adecuadamente en los diversos ámbitos de la sociedad. Esta dimensión del concepto pone en primer plano los fines atribuidos a la acción educativa y su concreción en los diseños y contenidos curriculares.

Finalmente, una tercera dimensión es la que refiere a la calidad de los procesos y medios que el sistema brinda a los alumnos para el desarrollo de su experiencia educativa. Desde esta perspectiva una educación de calidad sería aquella que ofrece un adecuado contexto físico para el aprendizaje, un cuerpo docente adecuadamente preparado para la tarea de enseñar, buenos materiales de estudio y de trabajo, estrategias didácticas adecuadas, etc. Esta dimensión del concepto pone en primer plano el análisis de los medios empleados en la acción educativa. Según las opiniones de Elola y Toranzos (2000), las tres dimensiones del concepto son esenciales a la hora de referirse a la calidad de la educación.

Por otra parte, en opinión de Navarro (1997) la educación de calidad es la que logra resultados que permitan el progreso y la modernización. Elevar la calidad es entonces encontrar los medios necesarios para el logro de los fines, midiendo los resultados se adecuan los medios pertinentes.

De acuerdo con lo señalado por Arrien (1998), la calidad parece estar muy asociada a los procesos y resultados del desarrollo educativo del educando, desarrollo que se manifiesta en los aprendizajes relevantes del alumno como sujeto, haciendo que éste crezca y se desarrolle personal y socialmente mediante actitudes, destrezas, valores y conocimientos que lo convierten en un ciudadano útil y solidario.

Es así como en una visión global e integral, la calidad de la enseñanza es el resultado de un conjunto de procesos vinculados, de manera que para mejorar la calidad se debe analizar los procesos intermedios o coadyuvantes, en diversos grados de los aprendizajes y no sólo su resultado final.

Si bien los estudios al respecto evidencian que la calidad de la educación depende de múltiples factores, según los autores Bellei, Muñoz, Pérez y Raczynski (2003) existen elementos de gran incidencia en el resultado de aprendizaje de los alumnos entre ellas el proceso pedagógico y la interacción en el aula, y complementariamente la gestión de la escuela, la cual orienta la dirección de los procesos educativos haciéndolos más eficientes, administrando adecuadamente los recursos y guiando y motivando al recurso humano hacia el logro de aprendizajes en los estudiantes. 


\section{c. Situación de la Calidad de Educación en Chile.}

En Chile, a partir del año 1998, se comenzó medir la calidad de la educación mediante el Sistema Nacional de Medición de la Calidad de la Educación (SIMCE), el cual a través la aplicación nacional de pruebas estandarizadas evalúa el logro de los Objetivos Fundamentales y Contenidos Mínimos Obligatorios (OF-CMO) del Marco Curricular vigente en diferentes subsectores de aprendizaje. Con su implementación se evidenció la brecha significativa en la calidad de la educación, según el nivel socioeconómico de proveniencia de los niños y en especial los que estudian en establecimientos municipales.

Constantemente, en los resultados de las pruebas Simce es posible apreciar cómo los malos resultados se focalizan en los sectores socioeconómicos bajos y medio bajo, independiente de sí el sostenedor es un municipio o particular subvencionado ${ }^{48}$, siendo en algunos casos más bajo el puntaje en los colegios particulares subvencionados. Sin embargo, el problema radica en que el $40 \%$ de los estudiantes que rinde la Prueba Simce en $4^{\circ}$ básico, proviene de los sectores más pobres y de ellos el $75 \%$ asiste a un colegio municipal. ${ }^{49}$

Las mediciones Simce, desde el año 2003, han arrojado una diferencia promedio de 60 puntos entre los colegios municipales respecto de los colegios particular pagado y de 25 puntos promedio bajo los particular subvencionado, situación que se replica en los resultados de todas las pruebas estandarizadas, nacionales como la Prueba de selección Universitaria (PSU) o internacionales como TIMSS y PISSA.

Esto ha puesto de manifiesto la necesidad de mejorar la calidad de la educación entregada a los alumnos en el sistema público administrado por los municipios, en el cual se educan niños y jóvenes de sectores más vulnerables y con escaso capital cultural, por lo que además resulta mucho mayor el desafío de difícil enseñar y aprender.

Cabe señalar que la situación socio económica de los alumnos, por sí sola, no explica los malos resultados en los aprendizajes de los niños. Según Murillo, (2003); ..."una escuela eficaz promueve de forma duradera el desarrollo integral de todos y cada uno de sus estudiantes más allá de lo que sería previsible teniendo en cuenta su rendimiento inicial y su situación social, cultural y económica". Es así como una escuela eficaz favorece el desarrollo de todos y cada uno de sus alumnos y éstos deben progresar más de lo que es dable esperar conforme a las características socioeconómicas y culturales de su familia"...Complementariamente, una escuela eficaz responde por el desarrollo integral del alumno, esto es, además de buenos resultados de aprendizaje, se preocupa de su formación en valores, bienestar

48 Resultados Nacionales Simce 2006, 2007, 2008 y 2009 (para $4^{\circ}$ básico.

49 Fuentes, Ricardo. Calidad de la Educación. Ministerio de Educación. 
y satisfacción, desarrolla toda la personalidad de los alumnos.

En este sentido, los establecimientos municipales presentan un conjunto de debilidades que es producto, entre otros factores, del empobrecimiento y falta de financiamiento generado por la disminución de las matrículas, por tanto de los recursos de subvención, de las trabas administrativas en la gestión de recursos humanos y materiales, de la falta de rendición de cuentas públicas, difusa definición de atribuciones y escasa responsabilidad por los resultados; que inciden en la calidad de la educación que entregan.

Por esto la gestión de la Dirección de Administración de Educación Municipal cumple un rol clave al momento de mejorar la calidad de la educación que entrega a los alumnos de los sectores más vulnerables, ya que en su rol de sostenedor de la educación pública, enfrentan un conjunto de problemáticas caracterizadas por la falta de claridad en los roles, funciones que deben desarrollar y en las atribuciones que tienen para cumplirlos que repercuten en las orientaciones y gestión de los establecimientos educacionales bajo su responsabilidad.

\section{d. Funcionamiento y Gestión de las Direcciónes de Administación de} Educación Municipal

Respecto a las funciones que les competen a los DAEM nos encontramos con distintas normativas complementarias, pero también divergentes entre sí, en las cuales se le entregan roles y atribuciones o bien se alude a los municipios sobre materias de educación. De la revisión realizada sobre el conjunto de normativas, documentos, planes y otros es posible observar al menos cinco roles específicos:

\section{Sostenedor y Administrador:}

Las funciones como sostenedor están fijadas en el DFL $\mathrm{N}^{\circ} 2$ (Ley $\mathrm{N}^{\circ}$ 19.602) de 1998, que fija la nueva forma de asignación de subvenciones. En ella se indica que cuando la administración de la educación sea asumida por el Municipio, éste deberá nombrar especialmente a una persona encargada de la educación que asumirá la calidad de "sostenedor" con los derechos y deberes que éstos tienen. Dando origen así a las Direcciones de Administración de Educación Municipal que tienen carácter de sostenedor, ya que anterior a esta ley los municipios entregaban los servicios educacionales adosados a otras unidades, o direcciones, o en unidades creadas con mayores atribuciones.

A partir de esta ley que crea las Direcciones de Educación Municipal se incorpora la educación a la gestión de los municipios, además se define la necesidad de contar con un sistema propio de administración dentro del Municipio.

Posteriormente, la ley de Subvención Especial Preferencial (Ley SEP) confiere mayores atribuciones a los sostenedores, municipales y particulares subvencionados, en la gestión de calidad de los establecimientos, mediante el cumplimiento de los Planes de Mejoramiento Educativo y la rendición de cuentas públicas. 


\section{Organismo Municipal:}

Dentro de las principales finalidades del Municipio está "satisfacer las necesidades de la comunidad local y asegurar su participación en el progreso económico, social y cultural de las respectivas comunas" ${ }^{\prime 50}$, y dentro de sus funciones compartidas la ley $n^{\circ} \mid 8.695$ establece que..." "las municipalidades en el ámbito de su territorio podrán desarrollar, directamente o con otros órganos de la administración del Estado, funciones relacionadas con la educación y la cultura".

En este contexto, los Municipios al entregar servicios de educación están comprometidos con el aporte de estos servicios al desarrollo comunal y territorial, el cual queda indicado en la ley $19.410^{51}$. En este documento se establece que los Municipios, a través de las Direcciones de Educación Municipal, deben elaborar un Plan de Desarrollo Educativo Municipal (PADEM), el cual es presentado para su aprobación al Concejo Municipal.

En este sentido se reconoce el rol de Organismo Municipal que tienen las direcciones de Administración de Educación Municipal y, por tanto, adhieren a los principios básicos de los municipios sobre el desarrollo territorial y la satisfacción de necesidades de sus habitantes, como también de sus características organizativas y políticas.

\section{Rol de Institución Pública y Estatal:}

La Dirección de Administración de Educación Municipal, por tener un carácter de Organismo Público (municipal), está regida por la Ley $N^{\circ} 18.575$, Ley Orgánica Constitucional de Administración del Estado. Al estar descentralizado actúa bajo una personalidad Jurídica determinada, con bienes y recursos fiscales del Municipio; sin embargo, ésta afecta a las normativas, reglamentos y dictámenes a los cuales están sujetos los recursos fiscales. Esto implica un sistema de administración engorroso y rígido que muchas veces dificulta la gestión, ya sea por lo complejo de los procedimientos y normativas y/o por la falta de personal competente para ejercer esta función.

Si bien los recursos provenientes de la subvención pública se administran en cuentas separadas de las del Fondo Común Municipal, su uso está sujeto a todas las normativas vigentes para el sector público. En este sentido, las funciones del DAEM en torno a este rol son a lo menos las siguientes:

50 Ley Constitucional de Municipalidades.

51 Modifica la ley $n^{\circ} 19.070$, sobre estatuto de profesionales de la educación, el decreto con fuerza de ley $n^{\circ} 5$, de 1993, del Ministerio de Educación, sobre subvenciones a establecimientos educacionales, y otorga beneficios que señala. 
- Proponer y ejecutar medidas tendientes a materializar acciones y programas relacionados con salud pública y educación y otros servicios incorporados a su gestión.

- Administrar los recursos humanos, materiales y financieros de tales servicios, en coordinación con la Unidad de Administración y Finanzas.

En el año 2008 fue publicada la ley $N^{\circ}$ 20.248, que establece la ley de Subvención Escolar Preferencial (SEP), que está destinada al mejoramiento de la calidad de la educación de los establecimientos educacionales subvencionados que se impetrará por los alumnos prioritarios del primer y segundo nivel de transición $n^{52}$, y sus recursos deben ser administrados también bajo esta modalidad, en tanto los establecimientos Particulares Subvencionados utilizan sus propio mecanismos para la contratación de personal, compra de insumos y productos, adquisición de bienes, entre otros.

\section{Rol de Empleador:}

En el Estatuto Docente dictado en 1980 y posteriormente modificado en la Ley 19.070 de 199I se establece el traspaso del personal de los servicios de educación a los municipios independiente de la Planta Profesional. Complementariamente, el año 1991 se promulgó el Estatuto Docente, que establece las negociaciones centralizadas de salarios y la inamovilidad de los docentes, independientes de evaluaciones de desempeño, lo que ha generado una situación de rigidez en el sistema. Adicionalmente se establecen como funciones de la Dirección de Administración de Educación Municipal la definición y adecuación de la dotación docente, por tanto pueden realizar la contratación y traslado de profesores en las escuelas de la comuna. Desde 1996 se incorpora el Sistema Nacional de Evaluación de Desempeño Docente, que entrega a las escuelas información sobre procesos y resultados educativos y establece incentivos a los profesores. Dentro de este programa se inserta la Ley de Evaluación Docente, que mediante un proceso nacional califica el desempeño pedagógico de los profesores en cuatro niveles: destacado, competente, básico e insatisfactorio, estableciendo un sistema de capacitación desde el nivel central para los profesores con baja calificación.

También se hace la diferenciación respecto de los profesionales docentes regidos por el Estatuto Docente, los profesionales de apoyo a la gestión educativa que son funcionarios públicos (municipales) y los no docentes de apoyo en las escuelas regidos por la legislación laboral vigente.

Esta situación genera un complejidad en la gestión del Recurso Humano ya que el universo de personas administradas por la Dirección de Administración de Educación Municipal está regido por tres normativas distintas según su calidad funcionaria (docente, no docente y funcionarios municipales).

52 Corresponde a alumnos vulnerables, cuya situación socioeconómica dificulta su proceso educativo. 


\section{Rol de Gestión Técnico- Pedagógica:}

Según lo establecido en el DFL-I; DFL-I-3063 de 1980, sobre el manejo del Fondo Común Municipal, en lo que se refiere al traspaso de servicios del Sector Público al municipio, se indica que el servicio educativo estará sujeto a la supervigilancia técnica y fiscalización que disponga la ley, de parte de las entidades y servicios especializados, en este caso, al Ministerio de Educación a través de la Dirección Provincial de Educación.

Es así como también se establece en la ley 19.410 que el Plan de Desarrollo Educativo Municipal deberá ser enviado al Departamento Provincial de Educación para su revisión y observaciones. Estos departamentos provinciales son organismos desconcentrados funcional y territorialmente de las Secretarías Regionales Ministeriales, siendo entonces los que deben promover la mejora en la calidad de la educación.

Con el fin de poder articular e implementar adecuadamente los servicios educacionales los municipios han establecido reglamentos de funcionamiento de la Dirección de Administración de Educación Municipal. Al revisar las páginas WEB de 16 distintos municipios ${ }^{53}$, se observó que dentro de sus dentro sus funciones compartían las siguientes : 


\section{Cuadro $n^{\circ}$ I: Tipificación de la gestión de la Dirección de Administración de Educación Municipal}

\begin{tabular}{|c|c|}
\hline Tipo de rol & Principales funciones definidas \\
\hline $\begin{array}{l}\text { Sostenedor y } \\
\text { administrador }\end{array}$ & $\begin{array}{l}\text { - Cautelar que, en los establecimientos educacionales de su jurisdicción, se cumplan } \\
\text { todos y cada uno de los requisitos establecidos para mantener la calidad de } \\
\text { sostenedor de la función educacional del Estado. } \\
\text { - Controlar el cumplimiento del calendario escolar regional, en lo referido a los } \\
\text { establecimientos que están bajo su jurisdicción. } \\
\text { - Cautelar la existencia de la documentación oficial en los establecimientos } \\
\text { educacionales y en la Dirección de Administración de Educación Municipal } \\
\text { - Hacer cumplir al personal directivo de los establecimientos de la comuna las } \\
\text { normas técnico-pedagógicas emanadas del Ministerio de Educación. } \\
\text { - Coordinar con organismos del Ministerio de Educación (Secretaría Regional } \\
\text { Ministerial y/o Direcciones Provinciales de Educación), los programas relativos a } \\
\text { actividades extraescolares y culturales. } \\
\text { - Elaboración y cumplimiento de Planes de Mejoramiento Educativo de las escuelas } \\
\text { y liceos. }\end{array}$ \\
\hline $\begin{array}{l}\text { Organismo } \\
\text { Municipal }\end{array}$ & $\begin{array}{l}\text { - } \quad \text { Elaborar anualmente el Plan de Educación Municipal y la dotación docente } \\
\text { - } \quad \text { Asesorar al Alcalde en materias educacionales municipales ante el Ministerio de } \\
\text { - } \quad \text { Preparar informes periódicos de gestión educativa para ser presentados al } \\
\text { Ministerio de Educación, a la Alcaldía y/o al Concejo. } \\
\text { - } \quad \text { Colaborar en los programas y actividades de Extensión y Cultura. } \\
\text { - Integrar el Comité de Emergencia. }\end{array}$ \\
\hline $\begin{array}{l}\text { Institución Pública } \\
\text { y Estatal }\end{array}$ & $\begin{array}{l}\text { - Procurar el mejoramiento de la infraestructura escolar y establecer normas para } \\
\text { su mantenimiento y su uso para fines educativos. } \\
\text { - } \quad \text { Presentar, para su aprobación a los organismos pertinentes, anteproyectos para } \\
\text { reparaciones mayores, ampliaciones y reposiciones de locales escolares. } \\
\text { - Cumplir con las normas de la Ordenanza General de Construcciones y con las } \\
\text { especificaciones técnicas del Ministerio de Educación en lo que se refiere a locales } \\
\text { escolares. } \\
\text { - Velar por el cumplimiento de la Ley de Subvenciones. } \\
\text { - Velar por la utilización eficiente de los recursos del Ministerio de Educación. }\end{array}$ \\
\hline Empleador & 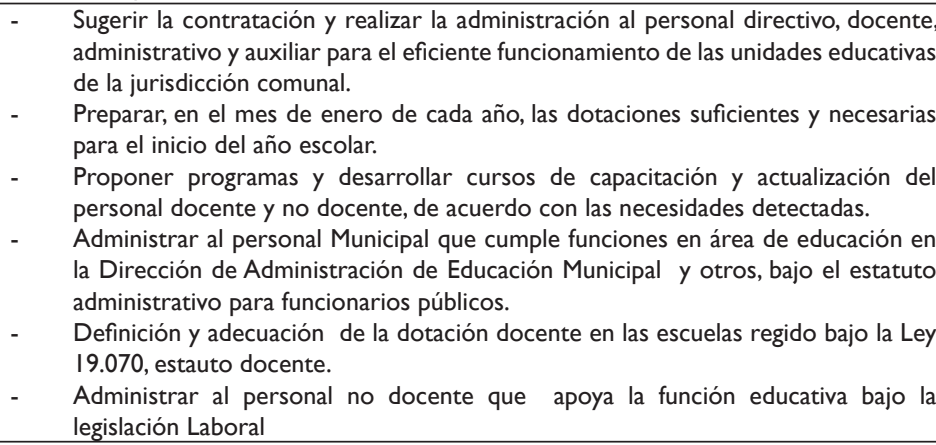 \\
\hline $\begin{array}{l}\text { Gestión técnico- } \\
\text { pedagógica: }\end{array}$ & $\begin{array}{l}\text { - Procurar la entrega de mobiliario y del material didáctico necesario y adecuado } \\
\text { para contribuir a mejorar la calidad del proceso educativo considerando las } \\
\text { orientaciones técnicas del Ministerio de Educación. } \\
\text { Instruir a los jefes de establecimientos educacionales en el sentido de dar las } \\
\text { facilidades del caso a los funcionarios de los distintos niveles del Ministerio de } \\
\text { Educación para el cumplimiento de sus actividades supervisoras, poniendo a su } \\
\text { disposición los antecedentes que éstos requieran. } \\
\text { Cautelar la aplicación de los programas complementarios que establezca el } \\
\text { Ministerio de Educación. } \\
\text { Propender y facilitar la ejecución de proyectos y programas de mejoramiento de la } \\
\text { calidad de la educación del Ministerio. }\end{array}$ \\
\hline
\end{tabular}

Fuente: Elaboración propia 
La situación de gestión de las Direcciones de Administración de Educación Municipal presentada en este artículo hace reflexionar sobre sí estas unidades, en tanto sostenedores de establecimientos educacionales municipales, tienen los elementos para dar solución a problemas estructurales y funcionales de la educación de su comuna y complementariamente sobre sí la problemática de la baja calidad de la educación que entregan a sus alumnos es abordada estratégicamente en su gestión.

\section{ELEMENTOS QUE CONDICIONAN LA GESTIÓNY FUNCIONAMIENTO DE LOS DAEM}

Dentro de la Planificación estratégica que desarrolla una organización existen dos conceptos centrales: Misión y Visión, que dan sustento a sus funciones, delimitan sus metas y plantean sus objetivos en el corto, mediano y largo plazo.

La Misión es entendida como un elemento central de la planificación estratégica, ya que mediante su formulación son establecidos los objetivos que guían el accionar de una organización. La misión es considerada como "el propósito general o razón de ser de la empresa u organización que enuncia a qué clientes sirve, qué necesidades satisface, qué tipos de productos ofrece $y$, en general, cuáles son los límites de sus actividades; por tanto, es aquello que todos los que componen la empresa u organización se sienten motivados a realizar en el presente y futuro para hacer realidad la visión de la empresa; esta misión se transforma en el marco de referencia que orienta las acciones, enlaza lo deseado con lo posible, condiciona las actividades presentes y futuras, proporciona unidad, sentido de dirección y guía en la toma de decisiones estratégicas" (Thompson y Strickland, 200 I).

Por su parte, la Visión es definida como "el camino al cual se dirige la empresa a largo plazo y sirve de rumbo y aliciente para orientar las decisiones estratégicas de crecimiento junto a las de competitividad", esto implica establecer un horizonte administrativo de amplias dimensiones, en el que se incluyen los diferentes ámbitos que precise una organización para dar respuesta a las necesidades de sus clientesusuarios.

\section{a. Misión}

Los datos entregados por los entrevistados indican que los DAEM han ido asumiendo funciones que, si bien no son atribuidas por ley, responden a requerimientos del sistema educativo actual.

Es así como la Misión que esbozan las Direcciones de Administración de Educación Municipal en opinión de las jefaturas entrevistadas, se vinculan a tres grandes ámbitos: el primero asociado al Apoyo Administrativo, el segundo al Apoyo Pedagógico y el último ligado a la Implementación de las Políticas Educacionales, como se presenta en el siguiente esquema: 
Esquema $n^{\circ}$ I: Misión de los DAEM

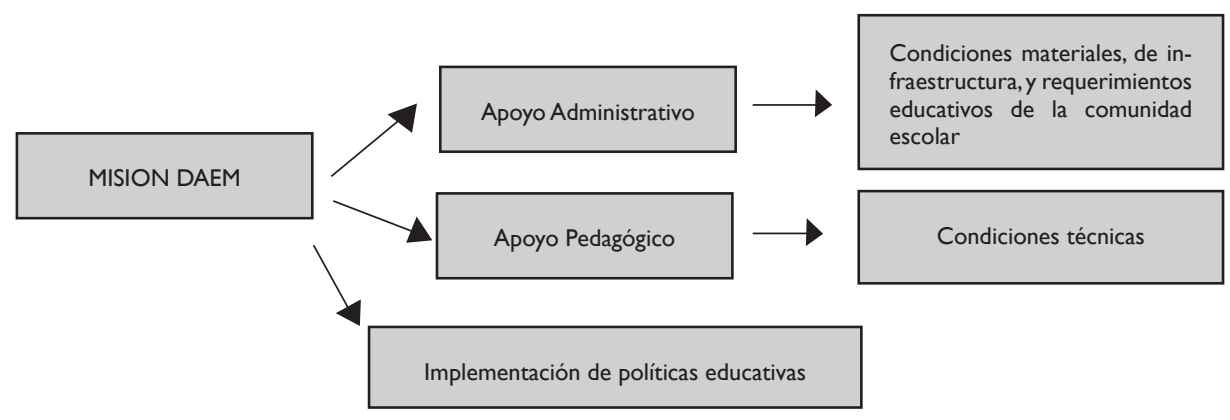

Del total de Jefes de Dirección de Administración de Educación Municipal entrevistados, existen tres que desarrollan una misión con relación a ejercer funciones de tipo administrativo, dos que definen su misión asociada al cumplimiento de las políticas educativas impulsadas por el Ministerio de Educación, mientras que las ocho comunas indagadas vinculan su trabajo al desarrollo de condiciones pedagógicas de infraestructura, equipamiento y materiales para lograr una educación de calidad en los establecimientos. Sólo uno de los entrevistados manifiesta desarrollar su misión con relación a las tres categorías formuladas.

Los DAEM que poseen una misión del tipo “Apoyo administrativo" enfatizan su función en la generación de condiciones materiales y de infraestructura pertinentes para una educación de calidad y la administración de los recursos financieros (subvención), tal como responder a requerimientos administrativos de los distintos actores involucrados en el proceso educativo (Mineduc, Directores, Apoderados, Alumnos, etc.), entregando respuestas a sus necesidades tanto administrativas como técnicas. Las comunas que manifestaron una misión de este tipo fueron Concepción, Coelemu y San Nicolás.

Por su parte los DAEM que presentan una misión relacionada con el "Apoyo pedagógico" ejecutan sus tareas con relación a la generación de condiciones de aprendizaje óptimas para una educación de calidad, entregando conocimientos y valores acordes a las directrices establecidas en el PADEM. Como se mencionó anteriormente, la totalidad de comunas consideradas en la muestra declara una misión vinculada a esta área.

Las Direcciones de Administración de Educación Municipal que reconocen una misión vinculada a la "Implementación de políticas educativas" velan por el mejoramiento de la calidad de la educación, planificando las acciones en el marco de los lineamientos generales implementados en la política educacional vigente, empleando enfoques de control y ajuste que permitan un buen desarrollo del sistema, siendo las comunas de Florida y San Nicolás las que se identifican con ésta.

Se percibe una diferencia entre la Misión percibida por los entrevistados y los roles y funciones que se les confiere por ley, especialmente en lo relacionado 
con las Políticas Educativas ya que si bien es percibido como un rol real en su gestión, éste no tiene un sustento legal que lo posibilite y está más bien vinculado a la autonomía Municipal, en la que cada municipio se ha organizado de la mejor forma según sus prioridades.

La Misión declarada por los Jefes de las Direcciones de Administración de Educación Municipal entrevistados se agrupa en tres áreas y no se percibe una función única ni específica para éstas, puesto que difieren entre municipalidades respecto de las prioridades y necesidades que tienen, según el tamaño y características de la comuna. No obstante todos los entrevistados visualizan el "Apoyo Pedagógico" como un elemento central para mejorar la calidad de la educación, enfatizando en diferentes actividades, a pesar de que no es una función obligatoria dentro de sus lineamientos, las que muchas veces se sobreponen con funciones de la Dirección provincial de Educación.

Es posible observar que para los entrevistados existe un rol de institución pública y municipal que tiende a priorizar los aspectos administrativos por sobre los resultados pedagógicos de la educación.

\section{b. Visión:}

La Visión planteada por los jefes de las Direcciones de Administración de Educación Municipal entrevistados se puede clasificar en tres tipos:

Relacionada con la satisfacción de necesidades sociales, que se presenta en seis de los Departamentos entrevistados:Talcahuano, Hualqui, Florida, Cabrero, Coelemu y San Nicolás y persigue entregar una formación integral de los alumnos, mediante un proceso de educación permanente.

Relacionada con la satisfacción de requerimientos institucionales, la cual se presenta en seis de las comunas: Concepción, Hualqui, San Pedro de la Paz, Florida, Coelemu y San Nicolás, y pretende ser un sistema capaz de generar condiciones operacionales eficientes, que permitan entregar una educación de calidad.

Y laVisión con relación al aporte para el desarrollo comunal. Que se presenta en tres de los establecimientos entrevistados: Talcahuano, Cabrero y San Nicolás y busca contribuir al desarrollo de la comuna con alumnos formados de manera integral, con el fin de aportar y servir a la comunidad en que viven, como se presenta en el siguiente esquema 
Esquema ${ }^{\circ} 2$ : Visión de los DAEM.

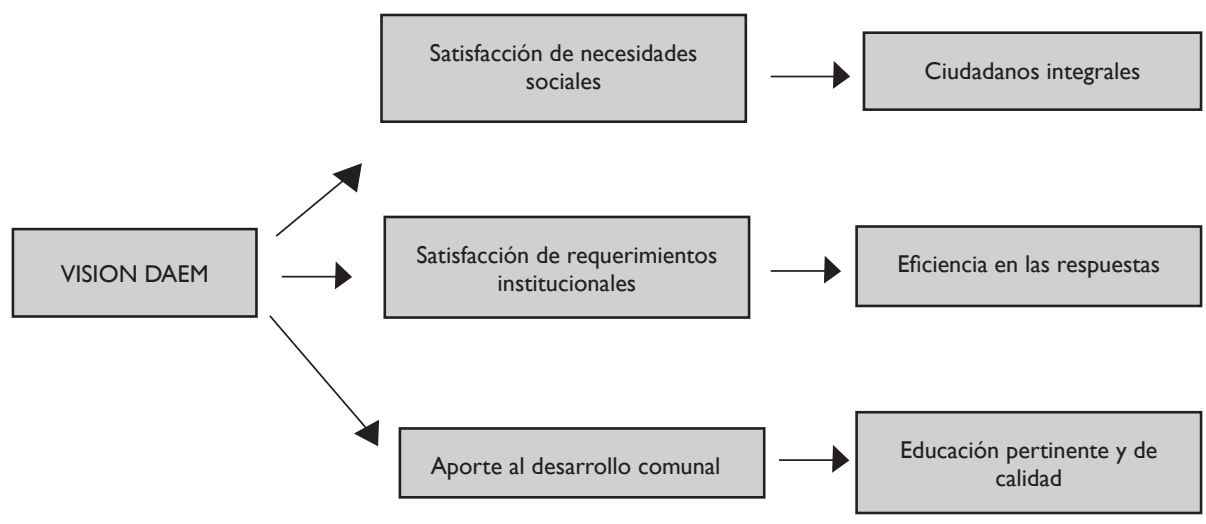

Los datos obtenidos señalan que las Direcciones de Administración de Educación Municipal proyectan su trabajo principalmente a los ámbitos de formación de ciudadanos integrales y de consolidación de un sistema educacional capaz de entregar respuestas eficientes a las necesidades educativas de sus establecimientos. En tanto, el desarrollo de una educación de calidad que incida en un aporte al desarrollo de las diferentes comunas es priorizado en menor medida, y sólo en uno de los casos estudiados la visión se desarrolla asociada a los tres ámbitos planteados.

En este sentido la Visión manifestada por los DAEM articula las necesidades educacionales con las necesidades para el desarrollo comunal, asumiendo su rol de Dirección Municipal. Sin embargo no existe una relación clara y vinculante entre la Visión y Misión percibida en las comunas investigadas, y de ésta con las funciones establecidas. Además, es importante destacar que dentro de los elementos declarados no se abordan los desafíos educacionales con relación al mejoramiento de la calidad en la educación entregada.

\section{c. Gestión}

Según el testimonio entregado por los entrevistados, es posible dividir el estilo de gestión en dos tipos:

Gestión vertical: es la gestión que se desarrolla de una manera piramidal, con alta jerarquización en el ejercicio de las funciones, las que se encuentran delimitadas por un organigrama institucional, el que define y coordina los roles que se ejercen. Bajo este modelo, las decisiones son tomadas de manera unilateral por la Jefatura del Departamento.

Gestión horizontal: la gestión se aborda mediante el Trabajo en Equipo, donde las decisiones son tomadas en conjunto con las diferentes unidades del Departamento. En este tipo de gestión cada Unidad del Departamento tiene sub-unidades a su 
cargo, en las cuales se replica la metodología del Trabajo en Equipo. Sin embargo, el Trabajo en Equipo vinculado a la toma de decisiones importantes se lleva a cabo principalmente con el área administrativa y de personal, y con el área técnica pedagógica, por lo que en ocasiones el Trabajo en Equipo se relega sólo al plano de la formulación y evaluación de proyectos por las unidades.

A continuación se presenta un esquema de estos estilos

Esquema $n^{\circ} 3$ : Estilos de gestión de los DAEM

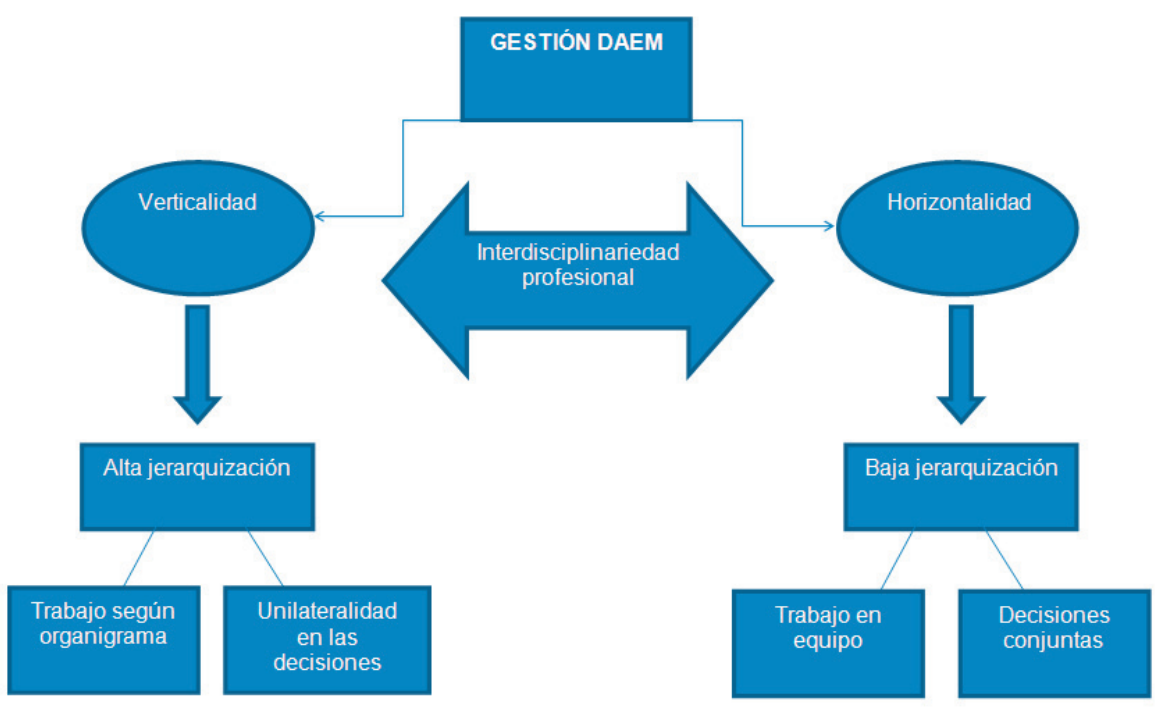

Si bien no existe una forma "pura" de estilo de gestión, las Direcciones de Administración de Educación Municipal de Concepción, Hualqui y San Pedro de la Paz se identifican con una gestión del tipo vertical, en tanto que Talcahuano, Florida, Cabrero, Coelemu y San Nicolás lo hacen con la del tipo horizontal, no existiendo relación entre el tamaño de la comuna y el tipo de gestión desarrollada por estos organismos.

En ambos modelos se trabaja coordinadamente con los demás organismos municipales, específicamente con la Alcaldía y Departamento de Planificación y Departamento de Obras, y con el Ministerio de Educación, a través de la Secretaría Ministerial y el Departamento Provincial de Educación.

Por su parte, la interdisciplinariedad profesional es percibida de vital importancia para el correcto ejercicio de las funciones de las Direcciones de Administración de Educación Municipal: tanto en aquellos con gestión vertical, como en los de gestión horizontal.

Si bien la mayoría de los entrevistados considera que el modelo horizontal es el óptimo y declaran su aplicación en la gestión de los DAEM, la realidad de su 
constitución de Organismo Público Municipal conlleva a una estructura altamente jerarquizada, con responsabilidades definidas por los diferentes roles, lo que no se contrapone con el trabajo en equipo real, pero establece el sistema de toma de decisiones.

Complementariamente es posible observar una segregación de las funciones en la cual el área de administración nuevamente tiene una mayor jerarquía por sobre las demás unidades. Adicionalmente, en el caso de municipios más grandes tienen un alto nivel de vinculación con otros organismos públicos pero segmentado, lo que dificulta la implementación de políticas integrales y sistémicas para resolver los problemas de calidad.

\section{d. Metas y objetivos}

Los Jefes de las Direcciones de Administración de Educación Municipal entrevistados indicaron que los objetivos y metas que poseen se encuentran sustentados en el Plan de Desarrollo de Educación Municipal (PADEM), un instrumento de planificación de la educación que debe ser elaborado por los municipios conforme con las exigencias establecidas en la Ley 19.410 de Educación de 1995 (DO 02.09.2006) en sus artículos $4^{\circ}, 5^{\circ}$ y $6^{\circ 54}$.

Este instrumento contiene los siguientes elementos dentro de su estructura:

- Un diagnóstico de la situación de los establecimientos municipales de la comuna, considerando aspectos académicos, extraescolares y administrativos.

- Una evaluación de la matrícula y asistencia media deseada y esperada en los establecimientos dependientes de la Municipalidad para el año siguiente y para los años posteriores.

- Las metas que el Departamento de Administración de Educación Municipal o que cada establecimiento pretendan alcanzar.

- La dotación docente y el personal no docente requeridos, fundados en razones técnico-pedagógicas.

- Los programas de acción por desarrollar durante el año en cada establecimiento y en la comuna. El presupuesto de ingresos, gastos e inversión para la ejecución del plan en cada establecimiento y en el conjunto de la comuna. 
Esquema $n^{\circ} 4:$ Metas y Objetivos de los DAEM

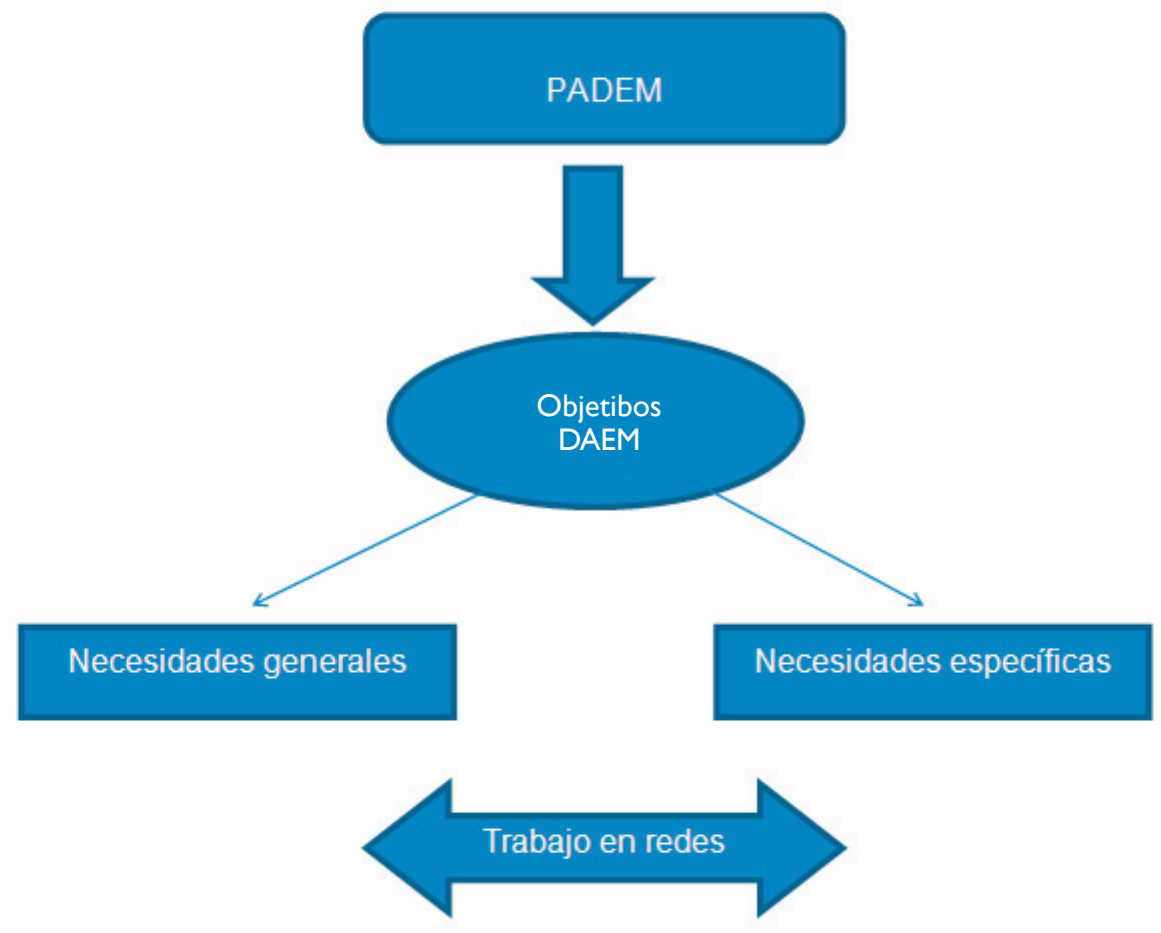

De las ocho jefaturas entrevistadas, sólo una declara metas y objetivos concretos, como por ejemplo, aumentar el puntaje de los resultados de la prueba SIMCE a 250 puntos, mejorar resultados en la evaluación docente, que es el caso de la comuna de San Pedro de la Paz. Las restantes hacen referencia al sustento, elaboración y gestión de las metas y objetivos, pero no los identifican de manera clara.

En este sentido, la mayoría de los Jefes de las Instituciones entrevistadas evidenció que sus metas y objetivos responden, en la práctica, a necesidades específicas que se van suscitando dentro del calendario académico (dotación de materiales específicos, reemplazos docentes), por lo que las necesidades generales, consideradas dentro del Plan de Desarrollo de Educación Municipal, en ocasiones quedan relegadas a un nivel teórico.

En todas las comunas indagadas se lleva a cabo un trabajo en redes con el sector público y/o privado, principalmente con universidades, con las cuales se abordan instancias de perfeccionamiento para los docentes y actividades de extensión para los alumnos. 
3. Problemas y debilidades que enfrentan las Direcciones de Administración de Educación Municipal

A partir de la opinión de las ocho jefaturas entrevistadas sobre la gestión y funcionamiento de estos organismos fue posible clasificar los problemas descritos en dos grupos: problemas estructurales, vinculados a dificultades en las políticas educativas y normativa vigente que no dependen de la acción del Municipio; y problemas funcionales, asociados a las diferentes debilidades en el ejercicio de roles y actividades de responsabilidad de los Departamentos de Administración de Educación Municipal .A continuación se presenta un análisis de cada uno de ellos y las posibles soluciones visualizadas por los entrevistados.

\section{a. Problemas estructurales.}

Dentro de los principales problemas estructurales, planteados por los entrevistados, encontramos seis situaciones

- Rigidez e inconsistencia del marco regulatorio, caracterizado por establecer limitantes en la gestión escolar y administrativa de los DAEM principalmente a causa de la burocracia del aparato estatal y su sistema de administración, y del Estatuto Docente, que restringe las posibilidades de cambio en los profesionales con mal desempeño, a lo que se suma una alta tasa de licencias médicas en docentes y resistencia a la jubilación básicamente por problemas económicos.

- Déficit presupuestario, como resultado del pago de subvención insuficiente para cubrir las necesidades educativas de los alumnos, especialmente los vulnerables, que además es cancelada por asistencia diaria, la disminución de la matrícula y el aumento en el gasto frente a las múltiples necesidades que presentan los Departamento de Administración de Educación Municipal. Ante esta debilidad se manifestó como solución la entrega de subvención por matrícula y no por asistencia, como se realiza actualmente, y que considere que para responder a las necesidades educativas de los sectores más vulnerables de la población, el costo es más elevado, por lo que requiere mayor inversión.

- Debilidad en la Formación Docente. Los resultados indicaron que se perciben pocas instancias de capacitación docente y problemas de focalización en ellas, a fin de dar respuesta a las verdaderas necesidades que enfrenta el sistema educativo actual, por lo que se requiere de capacitación pertinente a las debilidades encontradas en cada Departamento.

- Falta de innovación en educación. La incorporación de nuevas tecnologías, metodologías, y asimilación de nuevas realidades sociales es un dilema que enfrentan hoy en día las Direcciones de Administración de Educación Municipal, vinculado esencialmente a la mayor edad de los funcionarios y a las inadecuadas 
instancias de capacitación a las que acceden, pues no entregarían herramientas para enfrentar este tipo de debilidades.

- Alta diversidad y vulnerabilidad de los alumnos, que radica en problemas conductuales y de integración de los alumnos y la falta de manejo adecuado de estas situaciones complejas por parte de los docentes. Esta debilidad no tuvo propuesta de solución por parte de los entrevistados

Tabla $n^{\circ}$ I Análisis de Problemas Estructurales/ Soluciones Propuestas

\begin{tabular}{|c|c|}
\hline Problemas estructurales & Soluciones propuestas \\
\hline Rigidez e inconsistencia del marco regulatorio & Modificaciones al Estatuto Docente \\
\hline Déficit presupuestario & Pago de subvención por matrícula \\
\hline Formación y perfeccionamiento docente & $\begin{array}{c}\text { Capacitación docente acorde a requerimientos } \\
\text { actuales }\end{array}$ \\
\hline Falta de innovación en educación & Modificaciones a política de jubilación docente \\
\hline Alta diversidad y vulnerabilidad de los alumnos & \\
\hline
\end{tabular}

Al ser consultados sobre las soluciones a estas problemáticas indicaron que la Rigidez e inconsistencia del marco regulatorio podría ser abordada mediante modificaciones al Estatuto Docente; el Déficit presupuestario a través de la asignación de subvención por matrícula; la Formación y perfeccionamiento docente por medio de capacitaciones acotadas y acorde a los requerimientos actuales; y la falta de Innovación en educación, abordando modificaciones a la actual política de jubilación docente. A la problemática de Alta diversidad y vulnerabilidad de los alumnos no fueron planteadas soluciones por parte de las Jefaturas.

Con relación a lo anterior, de los principales problemas estructurales identificados, existen situaciones cuya solución no está en el ámbito de influencia de las Direcciones de Administración de Educación Municipal como la rigidez e inconsistencia del marco regulatorio, la disminución de matrícula, la formación y perfeccionamiento docente, y la falta de innovación educativa. Sin embargo, la ambigüedad y dispersión de las iniciativas normativas genera los espacios para que puedan desarrollar acciones que permitan abordar estas problemáticas localmente, pero al momento de identificar soluciones, las propuestas señaladas quedan fuera de su ámbito de gestión. Del mismo modo, existen problemas estructurales sobre los cuales los organismos Municipales perciben que no pueden generar acciones para su posible solución.

b. Problemas funcionales de las direcciones de administración de educación municipal

Dentro de los problemas funcionales evidenciados por los entrevistados y las respectivas soluciones propuestas, se encuentran cuatro situaciones: 
- Resultados de aprendizaje deficientes, que se asocia a bajos indicadores en mediciones estandarizadas, como es el caso de la Prueba SIMCE, en la que el nivel de logro obtenido por los establecimientos de las comunas investigadas sólo alcanza nivel inicial e intermedio ${ }^{55}$, y la Prueba de Selección Universitaria, donde los resultados no han sido alentadores en el tema: el primer año que se aplicó se presentaron a rendirla 30.000 postulantes menos que los años anteriores, la mayoría correspondiente a alumnos de la educación municipalizada, y luego de dos años de aplicación, el porcentaje de colegios municipalizados que ingresó a carreras en la Universidad de Chile bajó del 32\% en las antiguas pruebas a $25 \%$.

- Gestión administrativa deficiente, debido a problemas con la administración del personal, alta tasa de licencias médicas en funcionarios, bajo interés en participar de instancias de capacitación, y existencia de personal no capacitado para la administración, como es el caso de personal requerido para el manejo del portal Chilecompra.Ante esta problemática fue planteada como alternativa de solución la realización de capacitaciones para docentes y funcionarios administrativos de los DAEM

- Disminución de la matrícula. Uno de los problemas más serios que evidenciaron los Jefes entrevistados fue la abrupta disminución en la matrícula de los establecimientos que administran, que ha llevado en reiteradas ocasiones a dar cierre a escuelas a causa de la falta de alumnos, todo esto asociado a la estigmatización que sufre hoy en día la educación pública. Frente a esta debilidad, las Jefaturas entrevistadas consideran adecuado llevar a cabo las medidas pertinentes para retener a la matrícula actual, principalmente a través de la difusión de los elementos positivos de la educación pública.

- Debilidad en la Gestión Pedagógica, ligada a la inconsistencia de los sistemas evaluativos y de seguimiento-supervisión, que lleva al hecho de contratación de entidades externas para su realización. La respuesta manifestada por las Jefaturas entrevistadas fue la incorporación de instancias de evaluación y seguimiento de la gestión pedagógica.

- Problemas de convivencia escolar. La convivencia escolar es percibida como problema en la gestión de las Instituciones entrevistadas, causado esencialmente por la falta de integración entre la familia y la escuela.

55 Véase Simce on line: www.simce.cl

56 Véase: http://politicaspublicas.uc.cl/media/publicaciones/pdf/20100622214829.pdf 
Tabla n² Análisis Problemas Funcionales / Soluciones Propuestas

\begin{tabular}{|l|l|}
\hline \multicolumn{1}{|c|}{ Problemas funcionales } & \multicolumn{1}{c|}{ Soluciones propuestas } \\
\hline Resultados de aprendizaje deficientes & \\
\hline Gestión administrativa & $\begin{array}{l}\text { Capacitación para docentes y } \\
\text { funcionarios DAEM }\end{array}$ \\
\hline Disminución de la matrícula & Retención matrícula actual \\
\hline Debilidad en la gestión pedagógica & $\begin{array}{l}\text { Incorporar instancias de evaluación y } \\
\text { seguimiento a la gestión pedagógica }\end{array}$ \\
\hline Problemas de convivencia escolar. & \\
\hline
\end{tabular}

Es importante destacar que en las comunas rurales, además, son percibidos problemas de accesibilidad a los establecimientos y manejo metodológico de escuelas uni - docentes, para los cuales se proponen como posibles soluciones realizar visitas semanales a éstos, a fin de facilitar y mejorar la gestión, realizar convenios con Instituciones de Educación Superior para desarrollar prácticas en establecimientos rurales, y entregar movilización especial para los alumnos de estas escuelas.

\section{CONCLUSIONES}

Existe evidencia de la crisis en la calidad de la educación en Chile, la cual afecta con mayor intensidad a los niños de sectores más pobres y vulnerables, atendidos prioritariamente a través de la educación pública, cuyo sostenedor es el Municipio.

Este organismo público presenta un conjunto de dificultades legales, administrativas, técnicas y financieras, en la implementación de adecuadas y pertinentes medidas de administración de los servicios educativos, como así también problemas en la conducción para la adecuada gestión pedagógica en el proceso de enseñanza aprendizaje de los alumnos, lo que se traduce en los malos resultados educativos evidenciados a través de las mediciones estandarizadas.

A partir de los datos obtenidos en el estudio se desprende que las actuales condiciones organizacionales, administrativas y legales, que condicionan la acción de las Direcciones de Administración de Educación Municipal, dificultan focalizar su gestión en la principal problemática, asociada a la calidad de la educación pública en Chile. No obstante, si bien estas situaciones condicionan la calidad de la educación en los colegios municipales, no la determinan. La gestión que las Direcciones de Administración de Educación Municipal logran implementar, la administración de los recursos y la gestión directiva y de los docentes parecieran hacer la diferencia en los resultados de aprendizaje de los alumnos que atienden.

Estos organismos municipales tienen al menos cinco roles claramente definidos, sobre los cuales deben estructurar su gestión y planificación estratégica. 
En el estudio es posible observar que las funciones prioritarias se centran en desarrollar las condiciones pedagógicas, entendidas como recursos de aprendizaje necesarios para el desarrollo del proceso educativo en los establecimientos de su dependencia, en desmedro de su rol de sostenedor y de Unidad Técnico Pedagógica, que brinda apoyo pedagógico e implementación de políticas educativas para mejorar la calidad de la educación en la comuna.

Lo anterior se observa principalmente en el hecho de no tener una misión claramente establecida en las leyes o decretos que los crean, y por tanto, el énfasis en la misión que implementan está dado por la orientación estratégica del equipo humano que constituye la Dirección de Administración de Educación Municipal en cada comuna. En este contexto, estas instituciones reconocen la importancia del problema de la calidad de la educación, pero no se establece como prioridad al interior de su gestión, lo cual se evidencia claramente al analizar la visión planteada por ellos en donde la calidad de la educación que reciben los niños no está expresada como elemento central.

Las Direcciones de Administración de Educación Municipal sustentan sus metas y objetivos en el Plan de Desarrollo Educativo Municipal, instrumento de planificación requerido por normativa. Si bien, en algunos de estos instrumentos se aprecia preocupación por abordar la problemática de la calidad de la educación, no existen metas y objetivos claros, evaluables y cuantificables, para medir el avance o retroceso en este tema, como política comunal, más allá del esfuerzo de cada colegio en particular realiza para abordarlo a través de estrategias como el plan de Mejoramiento exigido por la Ley de Subvención Preferencial (Ley SEP)

Resulta complejo abordar las problemáticas estructurales del los DAEM, como el déficit presupuestario, la falta de innovación educativa y la inconsistencia de los marcos regulatorios; debido, entre otros facrores, a que su condición y origen no se encuentra en el ámbito de influencia de estos organismos municipales. No obstante, la ambigüedad y dispersión de los marcos normativos generan espacios para abordar con propuestas locales algunas de estas problemáticas.

Por su parte, el manejo adecuado de las problemáticas funcionales, como la gestión administrativa, los bajos resultados de aprendizaje de los alumnos, la diversidad de la gestión pedagógica, la disminución de la matrícula, entre otras, tendría posibilidades de concretarse con una mejor gestion de los DAEM, permitiendo el mejoramiento de la educación pública chilena.

Todas las Direcciones deAdministración de Educación Municipal manifiestan la necesidad de contar con instancias evaluativas, vinculadas a los niveles de logro obtenidos. Sin embargo, no se desarrolla ni implementa una gestión educativa basada en resultados. Esta debilidad se observa aún más con el hecho de contratar a instituciones externas para que realicen la evaluación de los resultados.

A través del estudio es posible considerar que para mejorar la calidad de la educación pública es necesario desarrollar y fortalecer el rol de sostenedor y gestión pedagógica en las Direcciones de Administración de Educación Municipal, ya que al observar sus deficiencias en la gestión, resulta evidente que no solamente 
se debe apuntar a la calidad del Directivo, del Docente y la Gestión de la escuela por sí sola, obviando el contexto jerarquizado que tienen las escuelas públicas por su rol público y municipal.

Las dificultades para mejorar la calidad de la educación pública también están asociadas a los modelos de administración educacional y a los resultados de la gestión que establece el sostenedor (en forma participativa o no) sobre los cuales es capaz de evaluar su gestión. En el caso de los Departamentos de Educación Municipal esta función fundamental ha quedado relegada a la administración y gestión de recursos públicos y de recursos humanos. Es así como, en el contexto actual, el mejoramiento de la calidad de la educación, especialmente en los sectores más vulnerables, se hace imperioso fortalecer el rol de sostenedor que hoy en día tienen los DAEM, de los cuales depende la mayor parte de las escuelas con bajo nivel de logro.

La alta vulnerabilidad de la población que asiste a los establecimientos municipalizados, la complejidad en la administración, y la entrega de mayores atribuciones sin recursos financieros, requiere de políticas públicas diferenciadas, que consideren el rol del sostenedor público y nuevas formas de financiamiento, que contribuyan a dar respuesta a estas necesidades y a resolver los nudos críticos de las Direcciones de Administración de Educación Municipal.

El fortalecimiento del rol de sostenedor del DAEM debería permitir una adecuada administración y conducción pedagógica en las escuelas, como también la implementación de políticas educativas de calidad comunes en los establecimientos a su cargo haciéndose parte de los resultados en los aprendizajes de los niños y las medidas propuestas por los colegios.

Lo anterior implica al menos la ejecución de tres medidas sistémicas y complementarias entre sí:

- Generar una mayor pertinencia, flexibilización y articulación de la estructura normativa que hoy rige a la gestión de los DAEM.

- Fortalecimiento de la función de sostenedor de los DAEM, mediante la redefinición de su planificación estratégica de corto y mediano plazo, con objetivos y metas claramente establecidos, la generación de una estructura organizacional que entregue respuestas a las demandas mayor conducción y apoyo pedagógico, como a la implementación de políticas educativas.

- Desarrollo de un modelo de gestión educativa profesionalizante, que les permita el paso de actor con un rol pasivo frente a las políticas institucionales y demandas educacionales de los establecimientos, a un rol de actor protagónico en la conducción, implementación y seguimiento de políticas educativas locales con las cuales responder a las demandas de calidad.

En el estudio es posible visualizar al menos cuatro problemáticas básicas sobre las cuales coinciden los Directores entrevistados, que es necesario abordar para mejorar estructuralmente la calidad de la educación pública: mayores 
recursos para enfrentar eficientemente el proceso pedagógico de los alumnos más vulnerables, la flexibilización de las normativas de administración financiera y de recursos humanos para orientar la gestión hacia los resultados educativos, entregar las atribuciones para la conducción pedagógica de las escuelas por parte de los sostenedores públicos y mejorar la calidad de los docentes que ejercen en las escuela.

Existen iniciativas ministeriales que buscan mejorar la educación pública modificando la actual institucionalidad hacia instancias descentralizadas denominadas “Agencias públicas de educación local”, también de carácter local para impartir los servicios de educación pública. Más allá del tipo de institucionalidad que imparta la educación, reconociendo las debilidades y fortalezas del actual sistema y las oportunidades y amenazas del propuesto, los cambios deber abordar las problemáticas estructurales antes mencionadas, para generar un contexto en el cual se posible ejercer atribuciones y liderazgo hacia una gestión educativa centrada en los resultados de aprendizaje de los estudiantes, especialmente los más vulnerables.

\section{REFERENCIAS}

Arríen, Juan et.al. (1998). La educación y la reforma de la educación en cinco países centroamericanos. Programa de Promoción de la Reforma Educativa en América Latina y El Caribe, Fundación Ford.

Astin,Alexander (199|). ¿Por qué no intentar otras formas de medir la calidad? On line: www.anuies.mx/servicios/P_anuies/publicaciones/revsup/res078/txt5.html

Barber, Michael y Mourshed, Mona (2008).Cómo hicieron los sistemas educativos con mejor desempeño del mundo para alcanzar sus objetivos. Programa de Promoción de la Reforma Educativa en América Latina y El Caribe PREAL, $N^{\circ} 4 I$.

Belleï, Cristian et.al. (2003). Escuelas efectivas en sectores de pobreza: ¿quién dijo que no se puede? Proyecto Movilizando las Capacidades de la Escuela, Fundación Chile. Estudio de UNICEF y Asesorías para el Desarrollo.

Braslavsky, Cecilia Diez, Factores para una Educación de Calidad para Todos en el Siglo XXI REICE. Revista Iberoamericana sobre Calidad, Eficacia y Cambio en Educación, vol. 4, núm. 2e, 2006, pp. 84-I0I

Dante Contreras, Osvaldo Larrañaga Lorena Flores, Félix Lobato y Víctor Macías (2003). Políticas educacionales en Chile: vouchers, concentración, incentivos y rendimiento. Universidad de Chile, Departamento de Economía.

Cornejo, Rodrigo. El experimento educativo chileno 20 años después: una mirada crítica a los logros y falencias del sistema escolar. Revista Electrónica Iberoamericana sobre Calidad, Eficacia y Cambio en Educación.

Crosby, P. (1993). “Hablemos de calidad”. Madrid. McGraw Hill. 
Elola, N. y Toranzos, L. (2000) Evaluación Educativa: una aproximación conceptual. Buenos Aires, Julio. On line: www.oei.es/calidad2/luis2.pdf

Espínola,Viola. Autonomía escolar: factores que contribuyen a una escuela más efectiva. Banco Interamericano de Desarrollo, División de Programas Sociales, Departamento Regional de Operaciones.

Espínola, V. y Silva, M. Competencias del sostenedor para una efectiva gestión del mejoramiento educativo en el nivel local: una propuesta. Revista En foco educación. Instituto de Políticas Públicas, Universidad Diego Portales.

Fuentes, Ricardo. Calidad de la Educación. Ministerio de Educación.

Harvey, L y Green, D. (1993) Defining quality. Assessment and evaluation in Higher Education.

López, Pablo (2010). Variables asociadas a la gestión escolar como factores de calidad educativa. Estudios Pedagógicos XXXVI, NI. Universidad de Chile, Facultad de Ciencias Sociales, Departamento de Educación.

Marcel, M. y Tokman, C. (2005). ¿Cómo se financia la educación en Chile? Estudio de Finanzas Públicas, Gobierno de Chile, Ministerio de Hacienda, Dirección de Presupuestos.

Murillo, Javier (2003). El movimiento teórico-práctico de mejora de la escuela. Algunas lecciones aprendidas para transformar los criterios docentes. Revista Electrónica, Iberoamericana sobre calidad, eficacia y cambio en educación. Vol. I, N² 2 . On line: http://www.ice.deusto.es/RINACE/reice/vol In2/Murillo.htm

Navarro, J. et. al. (1997).La reforma educativa en América Latina:Temas, componentes e instrumentos.

Paredes, R. y Lizama, O. Restricciones, Gestión y Brecha Educativa en Escuelas Municipales. Pontificia Universidad Católica de Chile.

Thompson A. y Strickland A. (200I). Administración Estratégica, Conceptos y Casos. Editorial Mc Graw Hill, I Iva. Edición.

Valenzuela, J. et al. (20l0). Estándares e indicadores de desempeño de sostenedores en el sistema educacional público chileno. Informe final. Centro de Investigación avanzada en educación. Universidad de Chile, Pontificia Universidad Católica de Valparaíso. 